\title{
Case Report: Necrotizing Fasciitis due to Cryptococcus Infection in Non-HIV Non-Organ Transplant
}

\author{
Rbab Taha ${ }^{1 *}$, Mohamed Alama ${ }^{2}$, Hessa Aljhdali ${ }^{3}$, Ayaz khan ${ }^{1}$ and Reham Kaki ${ }^{1,4}$ \\ ${ }^{1}$ Department of Infection Diseases, King Abdulaziz Hospital, Jeddah, Saudi Arabia \\ ${ }^{2}$ Department of Internal Medicine, King Abdulaziz Hospital, Jeddah, Saudi Arabia \\ ${ }^{3}$ Anatomic Pathology Specialist and Teaching Assistant, Saudi Arabia \\ ${ }^{4}$ Department of Internal Medicine, Medical College, King Abdulaziz hospital, Jeddah, Saudi Arabia
}

*Corresponding author: Rabab Taha, Department of Infection Diseases, King Abdulaziz Hospital, Jeddah, Saudi Arabia

\begin{abstract}
We are presenting 67-year-old female, known to have diabetes for 10 years, not compliance to medication. Presented with 3 weeks history of isolated right lower limb and left thigh pain. Then she started to develop redness and swelling in same areas. She had also History of dry cough for one week. Admitted as a case of necrotizing fasciitis based on clinical laboratory and radiological features. She was treated empirically with vancomycin and meropenem and went for debridement twice. Histopathology and culture showed Cryptococcus neoformans. Investigations were done to rule out other immune system disorders. We started her on high dose intravenous fluconazole. Ten days later she developed multi organ failure and death. As a conclusion, Cryptococcus might affect non-HIV patients regardless of their immune system.
\end{abstract}

\section{Keywords}

Disseminated, Cryptococcosis, Necrotizing fasciitis, Apparently immunocompetent host

\section{Abbreviations}

AIDS: Acquired Immunodeficiency Syndrome; GM-CSF: Anti-Granulocyte-Macrophage Colony Stimulating Factor

\section{Introduction}

Cryptococcosis is a yeast infection that mainly affects immunocompromised patients [1]. It gets into human bodies by inhaling the fungal particle causing asymptomatic infection. Once patient's immunity declines, this infection gets disseminated into various organs of the human body [2].
The Meninges are one of the most favorable places for Cryptococcus to infect. Nonetheless any organ can be affected. Cutaneous infections occur in 10-20\%. It presents in various forms $[2,3]$. In rare occasions, cryptococcosis may cause necrotizing fasciitis and myositis [4-8].

Necrotizing fasciitis spreads through the muscle fascia, due to its scarce blood supply, causing damage to the muscle fascia and subcutaneous adipose tissue [9]. It is usually caused by a bacterial infection. It is Classified based on the bacterial pathogen to type 1 and 2 . Type one is polymicrobial - aerobic and anaerobic bacteria $[10,11]$, while type two is monomicrobial $[12,13]$ rarely, necrotizing fasciitis can be caused by cryptococcal infection [14].

We present a case of cryptococcal necrotizing fasciitis in an immunocompetent female.

\section{Case Report}

A 53-year-old female, house maid, known case of poorly controlled type 2 diabetes mellitus for 10 years. Presented to our hospital with a history of right lower limb \& and left thigh pain and bullae, for 3 weeks prior to admission.

Before presenting to our hospital, she sought medical care in a private primary care center. She was prescribed an antibiotic -not Known -, and analgesics. She did not improve.

Citation: Taha R, Alama M, Aljhdali H, khan A, Kaki R (2019) Case Report: Necrotizing Fasciitis due to Cryptococcus Infection in Non-HIV Non-Organ Transplant. Clin Med Rev Case Rep 6:271. doi. org/10.23937/2378-3656/1410271

Accepted: June 10, 2019: Published: June 12, 2019

Copyright: (C) 2019 Taha R, et al. This is an open-access article distributed under the terms of the Creative Commons Attribution License, which permits unrestricted use, distribution, and reproduction in any medium, provided the original author and source are credited. 
She was admitted to our facility on $14^{\text {th }}$ of March 2018. She had a history of dry cough for one-week, oral ulcers, odynophagia and generalized fatigability. She had no history of fever, loss of weight, loss of appetite, Night sweats, or rashes. No history of focal weakness, neck pain, photophobia or seizures. There was no history of joint pain, genital ulcers, abdominal pain or diarrhea.

She had no past medical history of malignancy, autoimmune disease or immunosuppressive disease. She was not on any immunosuppressive medications. She denied traveling or contact with animals.

On examination, the patient was afebrile, tachycardic, there was no lymphadenopathy, no mouth or genital ulcers, no neck stiffness, no focal weakness, chest exam revealed normal breath sounds bilaterally. No abdominal tenderness, rigidity or organomegalies.

She had right leg Bulla, the largest was in the anterior segment, there were two small ones in the medial aspect of the leg, they were hot, erythematous and tender. There was green pus discharge from the large bulla. On her right thigh, there was a black necrotic based ulcer with minimal pus discharge and tenderness.

After taking blood samples and swabs from her pus discharge for cultures, she was started on Vancomycin, Tazocin and clindamycin. She had WBC of $33 \mathrm{k} / \mathrm{uL}$ with neutrophil predominance, hemoglobin was $11 \mathrm{~g} /$ dl, platelet was $65 \mathrm{~K} / \mathrm{uL}$. Her coagulation profile was normal at presentation. CRP was 207, she had normal kidney function and electrolytes, and normal LFTs. Her chest X-ray (Figure 1) showed reticulonodular pattern bilaterally. HIV, HbsAg, HCV-Ab were negative, ANA was negative. IgG, IgM, IgA were within the normal range.

She was referred to General Surgery as a case of Necrotizing Fasciitis for urgent surgical debridement.

She was taken to the Operation Room; she underwent surgical debridement for her right lower limb. Tissue cultures and Histopathology samples were sent to the laboratory.

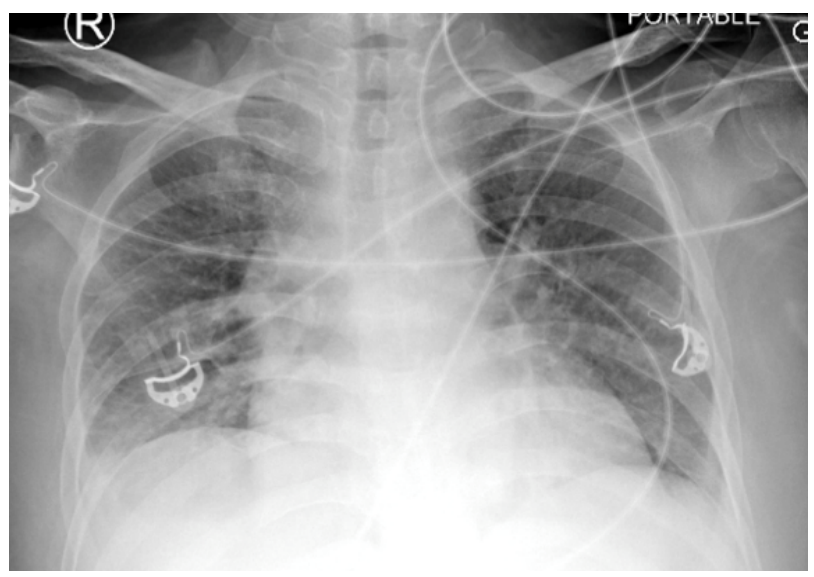

Figure 1: CT Leg report stated: "diabetic myonecrosis or developing necrotizing fasciitis".
After her OR she was Transferred to the Surgical ICU, Vacuum assisted wound closure device was applied on her wound. Her swab culture came back 1 day later, It grew ESBL E. coli. Her antibiotics regimen was changed based on the swab culture, Vancomycin and Tazocin were stopped, and meropenem was added.

Few days later the patient started to become hemodynamically unstable, she was taken back to OR,

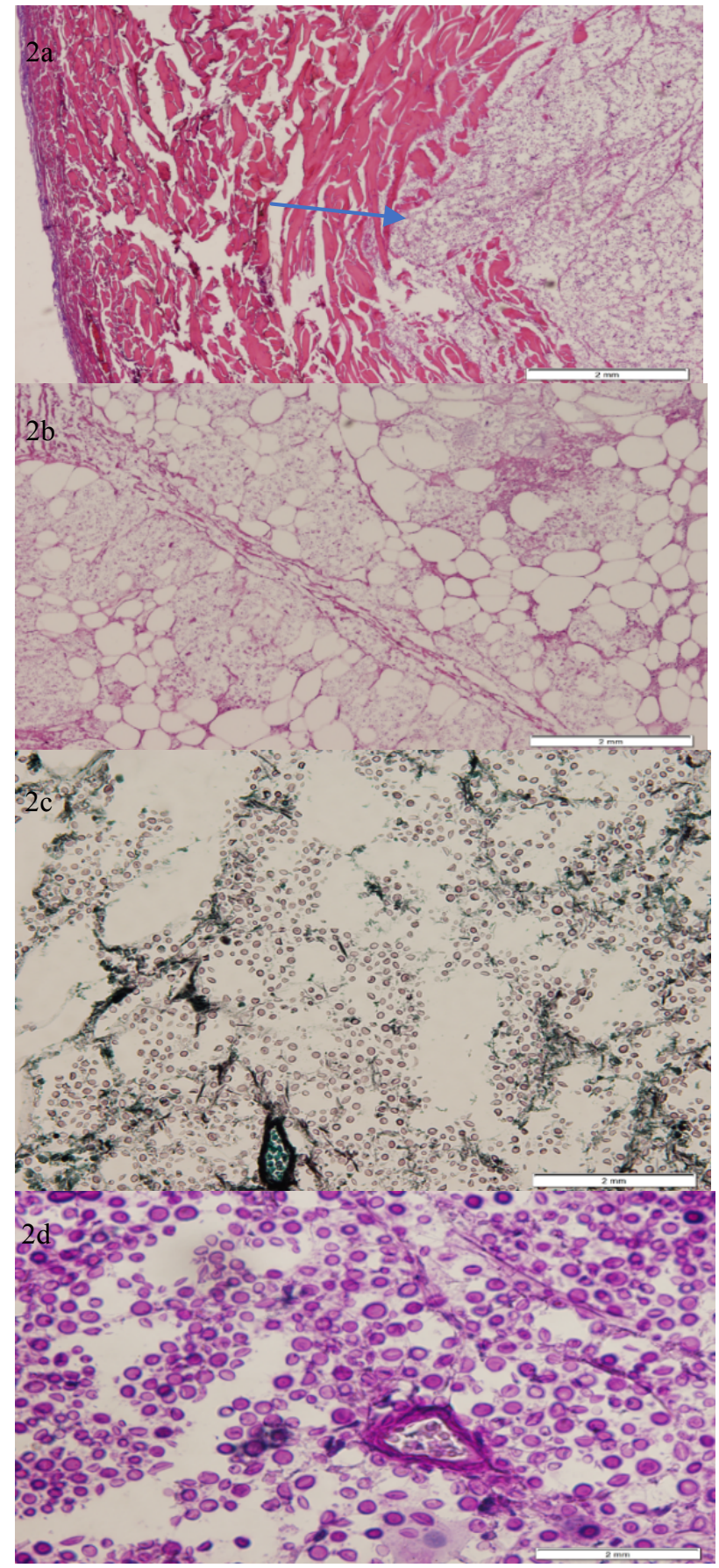

Figure 2: a) Histological section showing partially necrotic skin and subcutaneous tissue infested with fungal organisms identified by the arrow (H\&E, 4x); b) Necrotic subcutaneous tissue harbored by numerous round to oval in shape fungal yeast (H\&E, 10x); c) An encapsulated narrow-based budding Cryptococcal species that stain positively with Gomori methenamine silver stain (GMS, 20x); d) The fungal polysaccharide capsule stains bright red with mucicarmin stain (Mucicarmin stain, 40x). 
another session of right lower limb debridement was performed. On that day her Histopathology report was released stating Cryptococcus neoformans infection causing necrotizing fasciitis (Figure 2).

The patient was shifted back to Surgical ICU after her second right lower limb debridement, she was started on fluconazole. Unfortunately amphotericin B was not available at our hospital during that period, the patient and her family was offered right lower leg amputation as the optimal solution for the patient's current condition, but the patient and her family refused.

Few days later the patient's blood pressure started to drop, her platelet went down, her coagulation profile got prolonged. one day later she died from sepsis.

\section{Discussion}

Cryptococcus is an opportunistic infection more common in immunocompromised patients, including the patients who have impaired cell-mediated immunity. It is mainly seen in patients with AIDS [15]. It can also be diagnosed in non-HIV patients mainly those with hematologic malignancies, organ transplant recipients, patients on chronic corticosteroids or other immunosuppressive therapy like anti TNF agents [16]. Other possible conditions include history of alcoholism, collagen vascular disease, sarcoidosis and chronic organ failure [17]. A prospective study, published in 2010 derived from 23 center, it showed that Cryptococcus is the third most common fungal infection post candida and aspergillosis in solid organ transplant [18]. There are few published data regarding Cryptococcus in NonHIV, non-organ transplant. Although there are studies talking about diabetes and increase risk of infection due to inadequacy of immune system, it still is unusual to see this disease in diabetic patients. Some studies detected a deficiency in complement 4 in diabetic patients which is an important factor for opsonization which lead at the end to the lysis of the microorganism [19]. Increase glycation could inhibit the function of $T$ cell immunity which lead to interferon gamma (IFN- $\gamma$ ) and tumor necrosis factor (TNF)- $\alpha$ inhibition as well as the production of IL-10 by myeloid cells [20]. Cryptococcus might affect normal population regardless of their low immune system as there are few published cases regarding Cryptococcus infection in immunocompetent patients. There are only four studies since 1990s about necrotizing fasciitis with Cryptococcus in nonHIV patient's non-organ transplant patients. Periorbital necrotizing fasciitis due to Cryptococcus in young male published in 1990, he was immunocompetent with no underlying disease or history of steroid ingestion [21]. In 2008, a case published about 40-year-old male apparently immunocompetent, diagnosed as disseminated Cryptococci infection with necrotizing fasciitis who cured completely after combination therapy of surgical intervention and prolonged anti-fungal therapy [22].

Other 2 similar cases, were on steroid and cytotoxic medications [23]. The possible risk factors in apparently immunocompetent patient is temporary immune disorder like stress, using of steroid, uncontrolled diabetes and chronic kidney disease. other risk factor is immunoglobulin deficiency and granulocyte-macrophage colony stimulating factor (GM-CSF) antibodies. there was a study published in 2013, identified 7 non-HIV patients with Cryptococcus infection, all of them have GM-CSF autoantibodies [24].

Although there is not much studies about Cryptococcus in non-HIV patients, Data suggests presence of significant variation in clinical presentation and prognosis between HIV and non-HIV non organ transplant patient. A retrospective study published in 2013, showed that $88 \%$ of HIV Cryptococci infection associated with meningitis, $44 \%$ with blood stream infection, and only $12 \%, 4 \%$ with pulmonary and cutaneous infection respectively. While non HIV, non-organ transplant patients are more likely to develop pulmonary manifestations. $50 \%$ of apparently non-immunocompromised develop Cryptococcus meningitis, $40 \%$ pulmonary manifestations, $24 \%$ blood stream infections, and $5 \%$ bone and soft tissue infection. Same study suggests that 1-year mortality is: $26 \%$ in HIV patients, and $24 \%$ in organ transplant, while it was $35 \%$ in non-HIV, non-organ transplant patients, mostly due to delay in diagnoses [25].

There is well established treatment for Cryptococcus in HIV patients, while the treatment in immunocompetent patient depends on weak studies, due to fact that there are few studies conducted in this field [26].

\section{Conclusion}

The high index of suspicion for possible Cryptococcus infection is required not only in HIV patient but also in patients who might have defects in T-cell mediated immunity. More studies are needed to address the possible risk factors and modalities of treatment.

\section{References}

1. Banerjee U, Datta K, Majumdar T, Gupta K (2001) Cryptococcosis in India: The awakening of a giant? Med Mycol 39: 51-67.

2. Quartarolo N, Thomas I, Li H (2002) Cutaneous cryptococcosis. Acta Dermatol 11: 4.

3. Dharamshale SN, Patil SA, Gohil A, Chowdhary A, Oberoi C (2006) Disseminated cryptococcosis with extensive cutaneous involvement in AIDS. Ind J Med Microbiol 24: 228-230.

4. Gave AA, Torres R, Kaplan L (2004) Cryptococcal myositis and vasculitis: An unusual necrotizing soft tissue infection. Surg Infect 5: 309-313.

5. Bauza A, Redondo P, Rubio M (2005) Primary cutaneous cryptococcal cellulitis secondary to insect bite in an immunosuppressed patient after liver transplantation. Clin Exp Dermatol 30: 241-243. 
6. Hamann ID, Gillespie RJ, Ferguson JK (1997) Primary cutaneous cellulitis caused by Cryptococcus neoformans var. gattii in an immunocompetent host. Australas $J$ Dermatol 38: 29-32.

7. Hafner C, Linde HJ, Vogt T, Breindl G, Tintelnot K, et al. (2005) Primary cutaneous cryptococcosis and secondary antigenemia in a patient with long-term corticosteroid therapy. Infection 33: 86-89.

8. Sico JJ, Hughes E (2006) Necrotising cryptococcal vasculitis in an HIV-negative woman. Mycoses 49: 152-154.

9. Gozal D, Ziser A, Shupak A, Ariel A, Melamed Y (1986) Necrotizing Fasciitis. Arch Surg 121: 233-235.

10. Ellie JC Goldstein, Daniel A Anaya, E Patchen Dellinger (2007) Necrotizing Soft-Tissue Infection: Diagnosis and Management. Clinical Infectious Diseases 44: 705-710.

11. Wong $\mathrm{CH}$, Chang HC, Pasupathy S, Khin LW, Tan JL, et al. (2003) Necrotizing fasciitis: clinical presentation, microbiology, and determinants of mortality. J Bone Joint Surg Am 85: 1454-1460.

12. Miller LG, Perdreau-Remington F, Rieg G, Mehdi S, Perlroth $J$, et al. (2005) Necrotizing fasciitis caused by communityassociated methicillin-resistant Staphylococcus aureus in Los Angeles. N Engl J Med 352: 1445-1453.

13. Bisno AL, Stevens DL (1996) Streptococcal infections of skin and soft tissues. N Engl J Med 334: 240-245.

14. Capoor MR, Khanna G, Malhotra R, Verma S, Nair D, et al. (2008) Disseminated cryptococcosis with necrotizing fasciitis in an apparently immunocompetent host: A case report. Med Mycol 46: 269-273.

15. Bamba S, Lortholary O, Sawadogo A, Millogo A, Guiguemdé RT, et al. (2012) Decreasing incidence of cryptococcal meningitis in West Africa in the era of highly active antiretroviral therapy. AIDS 26: 1039-1041.

16. Pappas PG, Perfect JR, Cloud GA, Larsen RA, Pankey GA et al. (2001) Cryptococcosis in human immunodeficiency virus-negative patients in the era of effective azole therapy. Clin Infect Dis 33: 690-699.
17. Cancelli I, Merlino G, Serafini A, Valente M, Gigli GL (2008) Sarcoidosis as risk factor for cryptococcal meningitis in an apparently immunocompetent patient. Neurol Sci 29: 33-35.

18. Pappas PG, Alexander BD, Andes DR, Hadley S, Kauffman CA, et al. (2010) Invasive fungal infections among organ transplant recipients: Results of the Transplant-Associated Infection Surveillance Network (TRANSNET). Clin Infect Dis 50: 1101-1111.

19. Flyvbjerg A (2010) Diabetic angiopathy, the complement system and the tumor necrosis factor superfamily. Nat Rev Endocrinol 6: 94-101.

20. Price CL, Al Hassi HO, English NR, Blakemore Al, Stagg AJ, et al. (2010) Methylglyoxal modulates immune responses: Relevance to diabetes. J Cell Mol Med 14: 1806-1815.

21. Doorenbos-Bot AC, Hooymans JM, Blanksma LJ (1990) Periorbital necrotising fasciitis due to Cryptococcus neoformans in a healthy young man. Doc Ophthalmol 75: 315-320.

22. Malini R Capoor, Geetika Khanna, Rajeev Malhotra, Sunil Verma, Deepthi Nair, et al. (2008) Disseminated cryptococcosis with necrotizing fasciitis in an apparently immunocompetent host: A case report. Medical Mycology 46: $269-273$.

23. Tetsuya Hoshino, Kazuya Omura, Shinichi Kimura, Hiroyuki Takahashi, Katsuhiko Kamei, et al. (2017) A case of disseminated cryptococcosis with necrotizing fasciitis in a non-HIV patient. Acute Med Surg 4: 454-457.

24. Lindsey B Rosen, Alexandra F Freeman, Lauren M Yang, Kamonwan Jutivorakool, Kenneth N Olivier, et al. (2013) Anti-GM-CSF Autoantibodies in patients with Cryptococcal Meningitis. J Immunol 190: 3959-3966.

25. Pappas PG (2013) Cryptococcal infections in non-HIVinfected patients. Trans Am Clin Climatol Assoc 124: 61-79.

26. Sobel JD (2000) Practice guidelines for the treatment of fungal infections. For the Mycoses Study Group. Infectious Diseases Society of America. Clin Infect Dis 30: 652. 